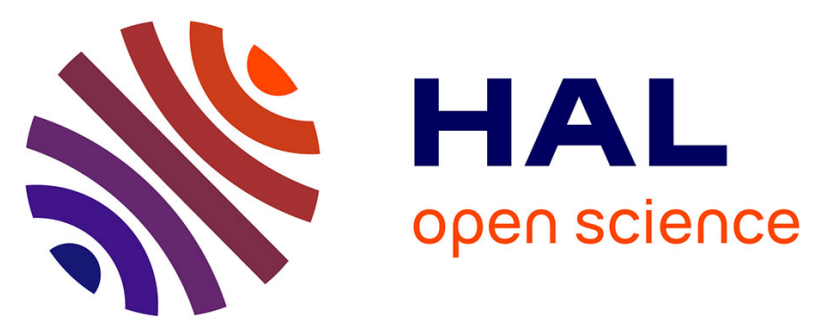

\title{
Stability and Stabilization of Aperiodic Sampled-Data Systems Subject to Control Input Saturation: A Set Invariant Approach
}

Daniel Denardi Huff, Mirko Fiacchini, Joao Manoel Gomes da Silva Jr

\section{- To cite this version:}

Daniel Denardi Huff, Mirko Fiacchini, Joao Manoel Gomes da Silva Jr. Stability and Stabilization of Aperiodic Sampled-Data Systems Subject to Control Input Saturation: A Set Invariant Approach. IEEE Transactions on Automatic Control, 2022, 67 (3), pp.142361429. 10.1109/TAC.2021.3064988 . hal-03188708

\section{HAL Id: hal-03188708 \\ https://hal.univ-grenoble-alpes.fr/hal-03188708}

Submitted on 1 Jul 2021

HAL is a multi-disciplinary open access archive for the deposit and dissemination of scientific research documents, whether they are published or not. The documents may come from teaching and research institutions in France or abroad, or from public or private research centers.
L'archive ouverte pluridisciplinaire HAL, est destinée au dépôt et à la diffusion de documents scientifiques de niveau recherche, publiés ou non, émanant des établissements d'enseignement et de recherche français ou étrangers, des laboratoires publics ou privés. 


\title{
Stability and Stabilization of Sampled-data Systems Subject to Control Input Saturation: a Set Invariant Approach
}

\author{
Daniel Denardi Huff, Mirko Fiacchini, and João Manoel Gomes da Silva Jr
}

\begin{abstract}
This work proposes a new method to deal with the stability analysis and stabilization of aperiodic sampled-data control systems subject to input saturation. An impulsive system representation is employed, with a linear flow and a nonlinear jump dynamics, in such a way that the evolution of the system at the sampling instants can be modeled by a difference inclusion defined by two set-valued maps. We show that to ensure the asymptotic stability it is sufficient to verify that a Lyapunov function decreases by a certain amount only at a grid of possible values for the sampling interval, as long as the increase of the function in continuous-time is conveniently bounded. Simulation results compare our approach with other ones.
\end{abstract}

Index Terms - sampled-data systems, input saturation, Lyapunov analysis

\section{INTRODUCTION}

$\mathbf{I}$ $\mathrm{N}$ many real-world applications, digital controllers are employed to control continuous-time plants. These sampled-data systems have been the objective of many research efforts in the last decades. The case of periodic sampling has been dealt with through several approaches (see [1], [2] for an overview or [3], [4] for the nonlinear case). Aperiodic sampling, on the other hand, is a more recent topic of study motivated by the implementation of networked control systems [5], which present fluctuations on the time between successive sampling instants.

In this context, different methods exist to study the stability of systems subject to a time-varying sampling interval. In [6], [7] the system is considered as affected by time-varying delay acting on the control input and its stability analysis is based on LyapunovKrasovskii functionals. Following similar ideas, in [8], [9] an approach using the so-called looped-functionals is presented. In [10], [11] the system is studied based on an uncertain discrete-time model that describes the evolution of the state at the sampling instants. Numerical tractable criteria for this approach can be obtained through the use of polytopic embeddings of the system transition matrix [12], [13]. Alternatively, norm-bounded approximations of this matrix can be used [14]-[16]. In [17], [18] a hybrid system approach is explored. For a general overview of those methods and other references, the reader can refer to the survey [19]. In particular, the stability of aperiodic sampled-data systems in the presence of control saturation is addressed, for instance, in [9], [20], considering a looped-functional approach, and in [21], where a set invariance approach based on differential inclusions is proposed.

This study was financed in part by the Coordenação de Aperfeiçoamento de Pessoal de Nível Superior - Brazil (CAPES) Finance Code 001 and by the French National Research Agency in the framework of the "Investissements d'avenir" program (ANR-15-IDEX02). J.M. Gomes da Silva Jr. is also supported by CNPQ, Brazil (grant $P Q-307449 / 2019-0)$.

D.D. Huff and M. Fiacchini are with Gipsa-lab, Université Grenoble Alpes (UGA), Grenoble, France. \{daniel.denardi-huff, mirko.fiacchini\}egipsa-lab.fr

D.D. Huff and J.M. Gomes da Silva Jr. are with the Grad. Program in Electrical Eng. (PPGEE) and the Dept. of Automation and Energy Systems (DELAE), Universidade Federal do Rio Grande do Sul (UFRGS), Porto Alegre, Brazil. \{daniel.huff, jmgomes\}@ufrgs.br
This work can be seen as an evolution of the results in [21], which presents a new approach to analyze the stability of aperiodic sampleddata systems subject to control saturation. Here an adaptation of the method allows not only the stability analysis of the nonlinear closedloop system but also provides a construtive way for designing a control law aiming at indirectly maximizing the region of attraction of the origin (RAO). Quadratic Lyapunov functions are employed, leading to stability and stabilization conditions in LMI (Linear Matrix Inequality) form. Unlike [21], which considers the static linear feedback of the system state, here we employ a more general control law: the control input can also depend linearly on its past value, resulting in a dynamic controller. In this case, the initialization of the controller can be seen as a control parameter and permits to improve the estimates of the RAO of the system.

To derive our results, an impulsive system representation is employed, with a linear flow and a nonlinear (due to the saturation term) jump dynamics. It is shown that the evolution of the system at the sampling instants can be modeled by a difference inclusion defined by two set-valued maps. From this setup, we show that to ensure the asymptotic stability it is sufficient to verify that the Lyapunov function decreases by a certain amount only at a grid of possible values for the sampling interval, as long as the increase of the function in continuous-time is conveniently bounded. The difference inclusion proposed here is different from the one in [21]. In particular, this new formulation allows to tackle the stabilization problem through convex optimization, which was not possible with the one in [21]. Furthermore, it is possible to consider quadratic (instead of piecewise quadratic) estimates of the region of attraction. The advantage of this is that direct criteria related to the size of the estimates can be considered, reducing the conservatism. Moreover, it is worth highlighting that this approach is different from the one in [12], [13], [22], for instance, where the decrease of the Lyapunov function between successive sampling instants is directly ensured for all possible sampling intervals through the use of polytopic embeddings of the system transition matrix.

The paper is organized as follows. In Section II an impulsive system representation for the aperiodic sampled-data system is presented. In Section III, we introduce the difference inclusions that model the evolution of the system state at the sampling instants. Section IV presents the stability analysis of the system and in Section V we show how to derive stabilization conditions from the previous results. A numerical example is provided in section VI. Some concluding remarks end the paper.

Notation. $\mathbb{R}$ is the set of real numbers and $\mathbb{N}$ the set of natural ones. We denote $\mathbb{N}_{n} \triangleq\{i \in \mathbb{N}: 1 \leq i \leq n\}$. For any function $f: \mathbb{R} \rightarrow \mathbb{R}^{n}$ we denote $f\left(t^{-}\right) \triangleq \lim _{\tau \mapsto t, \tau<t} f(\tau)$ if the limit exists and similarly for $f\left(t^{+}\right)$. The $\mathrm{i}$ - $t h$ row of the matrix $M$ is denoted $M_{(i)},\|\cdot\|_{p}$ denotes the $p$ norm. The maximal real part of the eigenvalues of matrix $A$ is denoted $\sigma_{\max }(A)$. The classical vector-valued saturation function is denoted $\operatorname{sat}(v): \mathbb{R}^{m} \rightarrow \mathbb{R}^{m}$, whose elements sat $(r)(v)$, for all $r=1, \ldots, m$, are

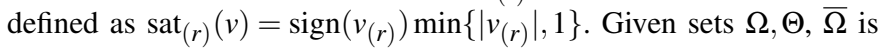
the closure of $\Omega$ and $\Omega \backslash \Theta \triangleq\{x: x \in \Omega, x \notin \Theta\}$. 


\section{Problem Formulation}

Consider the continuous-time plant described by the following linear model:

$$
\dot{x}_{p}(t)=A_{p} x_{p}(t)+B_{p} u(t)
$$

where $x_{p} \in \mathbb{R}^{n_{p}}$ and $u \in \mathbb{R}^{m}$ represent the state and the input of the plant, respectively. Matrices $A_{p}$ and $B_{p}$ have appropriate dimensions and are supposed to be constant.

We assume that the control signal is constrained in magnitude, i.e. $u(t) \in \mathscr{U}=\left\{u \in \mathbb{R}^{m}:\|u\|_{\infty} \leq 1\right\}$. Moreover, a sampled-data control policy is considered, i.e. the control signal is supposed to be computed from the values of system variables at sampling instants $t=t_{k}$, with $k \in \mathbb{N}$, and kept constant (by means of a zero order hold) for all $t \in\left[t_{k}, t_{k+1}\right)$. The difference between two successive sampling instants, given by $\delta_{k}=t_{k+1}-t_{k}$, is considered to be lower and upper bounded as follows

$$
0<\tau_{m} \leq \delta_{k} \leq \tau_{M}, \quad \forall k \in \mathbb{N} .
$$

The intersampling time $\delta_{k}$ can be variable, which allows to model an aperiodic sampling strategy. The particular case of periodic sampling corresponds to $\delta_{k}=\tau_{m}=\tau_{M}$ for all $k \in \mathbb{N}$. Thus, given the interval $\Delta=\left[\tau_{m}, \tau_{M}\right]$ with $0<\tau_{m} \leq \tau_{M}$ and $t_{0}=0$, the set of sequences of admissible sampling instants is defined as follows:

$$
\Theta(\Delta)=\left\{\left\{t_{k}\right\}_{k \in \mathbb{N}}: t_{k+1}=t_{k}+\delta_{k}, \delta_{k} \in \Delta, \quad \forall k \in \mathbb{N}\right\} .
$$

From the assumptions above, we consider in this work that the control law is generically given by:

$$
u(t)=\operatorname{sat}\left(K_{p} x_{p}\left(t_{k}^{-}\right)+K_{u} u\left(t_{k}^{-}\right)\right), \quad \forall t \in\left[t_{k}, t_{k+1}\right),
$$

i.e. the control input to be applied in the interval $\left[t_{k}, t_{k+1}\right)$ depends both on the sampled value of the state and the value of the control signal applied in the previous sampling interval $\left[t_{k-1}, t_{k}\right)$. It should be noticed that the control law considered in [21] corresponds to a particular case of (3), in which we have $K_{u}=0$.

Then, for every $\mathscr{T} \in \Theta(\Delta)$, the system dynamics can be represented by the following impulsive system:

$$
\begin{aligned}
& \left\{\begin{array}{l}
\dot{x}_{p}(t)=A_{p} x_{p}(t)+B_{p} u(t), \\
\dot{u}(t)=0,
\end{array}\right. \\
& \left\{\begin{array}{l}
x_{p}(t)=x_{p}\left(t^{-}\right)=x_{p}\left(t^{+}\right), \\
u(t)=u\left(t^{+}\right)=\operatorname{sat}\left(K_{p} x_{p}\left(t^{-}\right)+K_{u} u\left(t^{-}\right)\right),
\end{array}, \forall t \in \mathscr{T},\right.
\end{aligned}
$$

or, equivalently [23], by

$$
\begin{cases}\dot{x}(t)=A_{c} x(t), & \forall t \in \mathbb{R}_{+} \backslash \mathscr{T}, \\ x(t)=x\left(t^{+}\right)=A_{r} x\left(t^{-}\right)+B_{r} \operatorname{sat}\left(K x\left(t^{-}\right)\right), & \forall t \in \mathscr{T}, \\ x(0)=\left[x_{p, 0}^{\prime}, \operatorname{sat}\left(K_{p} x_{p, 0}+K_{u} u\left(0^{-}\right)\right)^{\prime}\right]^{\prime} \in \mathbb{R}^{n}, & \end{cases}
$$

where $x=\left[x_{p}^{\prime}, u^{\prime}\right]^{\prime} \in \mathbb{R}^{n}$, with $n=n_{p}+m$, is the extended system state and $A_{c}, A_{r} \in \mathbb{R}^{n \times n}, B_{r} \in \mathbb{R}^{n \times m}$ and $K \in \mathbb{R}^{m \times n}$ are given as follows

$$
\begin{gathered}
A_{c}=\left[\begin{array}{ll}
A_{p} & B_{p} \\
0 & 0
\end{array}\right], \quad A_{r}=\left[\begin{array}{ll}
I & 0 \\
0 & 0
\end{array}\right], \\
B_{r}=\left[\begin{array}{l}
0 \\
I
\end{array}\right], \quad K=\left[\begin{array}{ll}
K_{p} & K_{u}
\end{array}\right] .
\end{gathered}
$$

It should be noticed that, due to the saturation term, the closed-loop system (4) is nonlinear and the global stability of the origin cannot be a priori guaranteed. Moreover, if matrix $A_{p}$ is not Hurwitz, the global stabilization is actually impossible [24]. In this case, since the analytical characterization of the RAO is in general not possible, the idea is to estimate it through well-defined Lyapunov domains.

In this paper we are interested in the stability analysis and stabilization of the system (4) in a regional context, i.e. with determination of estimates of the RAO. The problems we focus on can therefore be stated as follows.

Problem 1 (Analysis): Given the bounds $\tau_{m}$ and $\tau_{M}$ on the intersampling time, provide conditions that allow to asses the local stability of the origin of the closed-loop system and to characterize estimates of the RAO.

Problem 2 (Design): Given the bounds $\tau_{m}$ and $\tau_{M}$ on the intersampling time, design the feedback gain $K$ in order to enlarge an estimate of the RAO of the closed-loop system.

\section{EQUIVALENT DISCRETE-TIME UNCERTAIN SYSTEM}

For a given initial condition and $\mathscr{T} \in \Theta(\Delta)$ the evolution of the state $x$ between two successive sampling instants, i.e. for $t \in\left[t_{k}, t_{k+1}\right)$, is continuous. Thus, since the dynamics is linear, it follows that:

$$
x(t)=e^{A_{c}\left(t-t_{k}\right)} x\left(t_{k}\right), \quad \forall t \in\left[t_{k}, t_{k+1}\right) .
$$

Hence, taking into account (5) and that $x\left(t_{k}^{-}\right) \neq x\left(t_{k}\right)$ (due to the impulsive control update, in fact, there is a discontinuity between $x\left(t_{k}^{-}\right)$and $x\left(t_{k}\right)$, see (4)), the dynamics between two successive sampling instants is given by the following discrete-time equation

$$
\begin{aligned}
x\left(t_{k+1}^{-}\right) & =e^{A_{c}\left(t_{k+1}-t_{k}\right)} x\left(t_{k}\right) \\
& =e^{A_{c}\left(t_{k+1}-t_{k}\right)}\left(A_{r} x\left(t_{k}^{-}\right)+B_{r} \operatorname{sat}\left(K x\left(t_{k}^{-}\right)\right)\right. \\
& =e^{A_{c} \delta_{k}} A_{r} x\left(t_{k}^{-}\right)+e^{A_{c} \delta_{k}} B_{r} \operatorname{sat}\left(K x\left(t_{k}^{-}\right)\right)
\end{aligned}
$$

where $\delta_{k}=t_{k+1}-t_{k} \in \Delta$. Thus, denoting $A(\delta)=e^{A_{c} \delta_{A}}, B(\delta)=$ $e^{A_{c} \delta} B_{r}$ and $x_{k}=x\left(t_{k}^{-}\right)$, the problem of stability and stabilization of the linear impulsive system (4) can be addressed by considering the following discrete-time nonlinear parametric uncertain system:

$$
x_{k+1}=A\left(\delta_{k}\right) x_{k}+B\left(\delta_{k}\right) \operatorname{sat}\left(K x_{k}\right), \quad \text { with } \delta_{k} \in \Delta .
$$

Consider now the partition of the interval $\left[\tau_{m}, \tau_{M}\right]$ in $J \in \mathbb{N}$ subintervals and define the set:

$$
\Delta_{J} \triangleq\left\{d_{j} \triangleq \tau_{m}+(j-1) \tau_{J}: j \in \mathbb{N}_{J}\right\}, \quad \tau_{J}=\frac{\tau_{M}-\tau_{m}}{J} .
$$

Since $\delta_{k} \in\left[\tau_{m}, \tau_{M}\right]$ and from (7), we have that for every $\delta_{k}$ there exist $d_{k} \in \Delta_{J}$ and $\tau_{k} \in\left[0, \tau_{J}\right]$ such that $\delta_{k}=d_{k}+\tau_{k}$ and it follows that:

$$
\begin{aligned}
& A\left(\delta_{k}\right)=A\left(d_{k}+\tau_{k}\right)=e^{A_{c}\left(d_{k}+\tau_{k}\right)} A_{r}=e^{A_{c} \tau_{k}} A\left(d_{k}\right), \\
& B\left(\delta_{k}\right)=B\left(d_{k}+\tau_{k}\right)=e^{A_{c}\left(d_{k}+\tau_{k}\right)} B_{r}=e^{A_{c} \tau_{k}} B\left(d_{k}\right) .
\end{aligned}
$$

Thus, from (6), we can write that:

$$
x_{k+1}=e^{A_{c} \tau_{k}} A\left(d_{k}\right) x_{k}+e^{A_{c} \tau_{k}} B\left(d_{k}\right) \operatorname{sat}\left(K x_{k}\right),
$$

with $d_{k} \in \Delta_{J}$ and $\tau_{k} \in\left[0, \tau_{J}\right]$. Then, given the value $x\left(t_{k}^{-}\right)=x_{k}$, the possible successors $x\left(t_{k+1}^{-}\right)=x_{k+1}$ are given by the difference inclusion

$$
x_{k+1} \in\left\{e^{A_{c} \tau}\left(A(d) x_{k}+B(d) \operatorname{sat}\left(K x_{k}\right)\right): d \in \Delta_{J}, \tau \in\left[0, \tau_{J}\right]\right\} .
$$

Hence, defining the following set-valued maps

$$
\begin{aligned}
& \mathscr{F}_{J}(z)=\left\{A\left(d_{j}\right) z+B\left(d_{j}\right) \operatorname{sat}(K z): d_{j} \in \Delta_{J}\right\} \subseteq \mathbb{R}^{n}, \\
& \mathscr{G}_{J}(y)=\left\{e^{A_{c} \tau} y: \tau \in\left[0, \tau_{J}\right]\right\} \subseteq \mathbb{R}^{n},
\end{aligned}
$$

for all $y, z \in \mathbb{R}^{n}$, then for some $y_{k} \in \mathscr{F}_{J}\left(x_{k}\right)$ we have that $x_{k+1} \in$ $\mathscr{G}_{J}\left(y_{k}\right)$, i.e. the dynamics in (8) is equivalently given by the difference inclusion

$$
x_{k+1} \in \mathscr{G}_{J}\left(\mathscr{F}_{J}\left(x_{k}\right)\right) .
$$

Thus, the difference inclusion system (10) permits to analyze the stability of the system (1) in closed loop with (3) under aperiodic sampling with inter-sampling time bounded by $\tau_{m}$ and $\tau_{M}$. 


\section{STABILITY ANALYSIS}

\section{A. Lyapunov setup}

Considering a Lyapunov function $V(x)$ and the difference inclusion in (10), a sufficient condition for the asymptotic stability of the origin of the system (1) in closed loop with (3) and the characterization of estimates of the RAO are given in the following theorem, where the sublevel sets of $V(x)$ are defined as

$$
\Omega_{c} \triangleq\left\{x \in \mathbb{R}^{n}: V(x) \leq c\right\}, \quad c \in \mathbb{R}
$$

Theorem 1: Let $V(x): \mathbb{R}^{n} \rightarrow \mathbb{R}$ be a differentiable positive definite Lyapunov candidate function. Let $\mathscr{D} \subseteq \mathbb{R}^{n}$ be a neighborhood of the origin. Suppose that there exist $c>0, \lambda \in(0,1)$ and $J \in \mathbb{N}$ such that $\Omega_{c}$ is bounded, $\Omega_{c} \subseteq \mathscr{D}$ and the following conditions hold:

a) $V\left(y_{k}\right) \leq \lambda V\left(x_{k}\right)$ for all $x_{k} \in \mathscr{D}$ and $y_{k} \in \mathscr{F}_{J}\left(x_{k}\right)$, with $\mathscr{F}_{J}(\cdot)$ as defined in (9);

b) $\dot{V}(x)<\alpha V(x)$ along the (nonzero) trajectories belonging to $\mathscr{D}$ of

$$
\dot{x}(t)=A_{c} x(t),
$$

with $\alpha=-\ln (\lambda) / \tau_{J}$. Then, the origin of the aperiodic sampleddata saturated control system composed by (1) and (3) is locally asymptotically stable. Moreover the set $\Omega_{c}$ is contained in the RAO.

Proof: Given $x_{k}=x\left(t_{k}^{-}\right) \in \Omega_{c}, x_{k} \neq 0$, we prove that $V\left(x_{k+1}\right)<$ $V\left(x_{k}\right)$ for all $x_{k+1}=x\left(t_{k+1}^{-}\right)$satisfying (10).

For each $x_{k+1} \in \mathscr{G}_{J}\left(\mathscr{F}_{J}\left(x_{k}\right)\right)$, there exist $d_{j} \in \Delta_{J}, y_{k} \in \mathscr{F}_{J}\left(x_{k}\right)$ and $\tau \in\left[0, \tau_{J}\right]$ such that $x_{k+1}=e^{A_{c} \tau} y_{k}$, where

$$
y_{k}=A\left(d_{j}\right) x_{k}+B\left(d_{j}\right) \operatorname{sat}\left(K x_{k}\right) \text {. }
$$

From condition a) we have that $V\left(y_{k}\right) \leq \lambda V\left(x_{k}\right)$. Moreover, notice that $x_{k+1}=e^{A_{c} \tau} y_{k}$ corresponds to the solution of (12) at time $t+\tau$ with initial condition $x(t)=y_{k}$. Then from condition $\mathrm{b}$ ) and since $\alpha=-\ln (\lambda) / \tau_{J}>0$ one obtains:

$$
\begin{array}{ll}
V\left(x_{k+1}\right)=V\left(y_{k}\right)<e^{\alpha \tau_{J}} V\left(y_{k}\right)=\frac{1}{\lambda} V\left(y_{k}\right) \leq V\left(x_{k}\right), & \text { if } \tau=0 \\
V\left(x_{k+1}\right)<e^{\alpha \tau} V\left(y_{k}\right) \leq e^{\alpha \tau_{J}} V\left(y_{k}\right) \leq V\left(x_{k}\right), & \text { if } \tau>0 .
\end{array}
$$

That is, $V\left(x_{k+1}\right)<V\left(x_{k}\right)$, for all $x_{k+1} \in \mathscr{G}_{J}\left(\mathscr{F}_{J}\left(x_{k}\right)\right)$. It follows that the state $x_{k}=x\left(t_{k}^{-}\right)$of the discrete-time system (6) converges to zero as $k \rightarrow+\infty$, provided that $x_{0} \in \Omega_{c}$.

Since the system dynamics is given by (5), it follows that

$$
x_{k+1}=x\left(t_{k+1}^{-}\right)=e^{A_{c}\left(t_{k+1}-t\right)} x(t), \quad \forall t \in\left[t_{k}, t_{k+1}\right)
$$

and, equivalently, we have

$$
x(t)=e^{A_{c}\left(t-t_{k+1}\right)} x_{k+1}, \quad \forall t \in\left[t_{k}, t_{k+1}\right) .
$$

Consequently,

$$
\|x(t)\| \leq \max _{\tau \in\left[0, \tau_{M}\right]}\left\|e^{-A_{c} \tau}\right\|\left\|x_{k+1}\right\|, \quad \forall t \in\left[t_{k}, t_{k+1}\right)
$$

and we can conclude that $x(t)$ is bounded in the interval $\left[t_{k}, t_{k+1}\right)$. Hence, since $x\left(t_{k}^{-}\right) \rightarrow 0$ as $k \rightarrow \infty$, we conclude that $x(t) \rightarrow 0$ as $t \rightarrow \infty$ and the origin of the aperiodic sampled-data saturating control system composed by (1) and (3) is asymptotically stable. Moreover, the set $\Omega_{c} \subseteq \mathscr{D}$ is contained in the RAO.

Fig. 1 gives a graphical interpretation of the reasoning implied by conditions a) and b) in terms of the behavior of the function $V(x)$, where we can see that $V\left(x_{k+1}\right)<V\left(x_{k}\right)$.

Remark 1: Since $\alpha$ is a positive scalar, condition b) in Theorem 1 does not impose a decreasing of the function $V(\cdot)$ in continuous-time. Actually, it ensures a bound on its potential increasing, which means that $A_{c}$ is not required to be Hurwitz. In fact, note that $A_{c}$ will never be Hurwitz because of its special structure including null eigenvalues. So the verification of condition $b$ ) will require a sufficiently large $\alpha>0$.

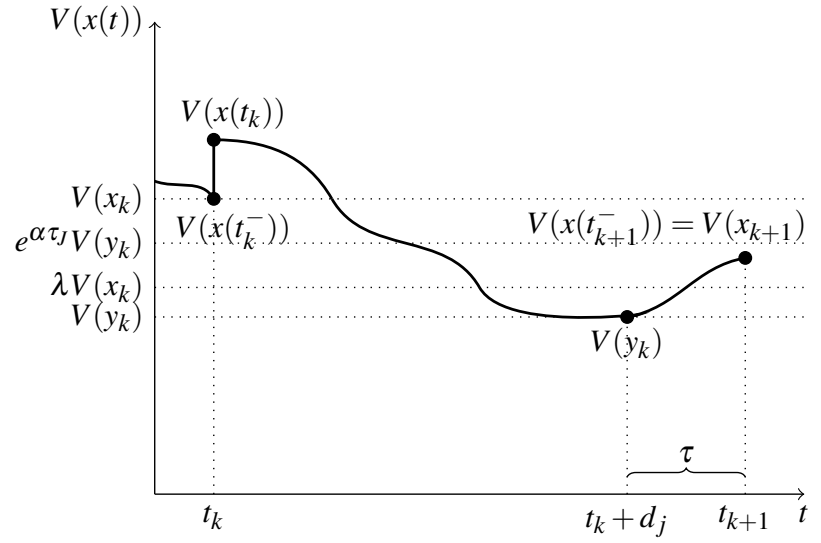

Fig. 1. Behavior of $V(x(t))$.

Remark 2: The estimate $\Omega_{c}$ of the RAO is related to $x_{0}=x\left(0^{-}\right)=$ $\left[x_{p}(0)^{\prime}, u\left(0^{-}\right)^{\prime}\right]^{\prime}$ where $x_{p}(0)=x_{p}\left(0^{-}\right)$is the initial plant state and $u\left(0^{-}\right)$is a free value that can be conveniently chosen to initialize the control such that $x_{0} \in \Omega_{c}$.

Theorem 2: Let $\mathscr{D} \subseteq \mathbb{R}^{n}$ be a bounded neighborhood of the origin. Suppose that the function $V(x): \mathbb{R}^{n} \rightarrow \mathbb{R}$ is continuously differentiable and positive definite, satisfying

$$
V\left(x_{k+1}\right) \leq \bar{\lambda} V\left(x_{k}\right)
$$

for all $x_{k} \in \mathscr{D}$ and for all $x_{k+1}$ given by (10), with $\bar{\lambda} \in(0,1)$. Assume that $V(\cdot)$ also satisfies

$$
\dot{V}(x)<\beta_{1} V(x), \quad \forall x \in \mathscr{N} \backslash\{0\}
$$

along the trajectories of (12), with $\mathscr{N}$ an arbitrary neighborhood of the origin and an arbitrary $\beta_{1} \in \mathbb{R}$. Then there exists $J \in \mathbb{N}, \lambda \in(0,1)$ such that items a) and b) of Theorem 1 hold.

Proof: Since $\mathscr{F}_{J}\left(x_{k}\right) \subseteq \mathscr{G}_{J}\left(\mathscr{F}_{J}\left(x_{k}\right)\right)$ for all $J \in \mathbb{N}$, it follows that

$$
V\left(y_{k}\right) \leq \bar{\lambda} V\left(x_{k}\right), \quad \forall x_{k} \in \mathscr{D}, \forall y_{k} \in \mathscr{F}_{J}\left(x_{k}\right) .
$$

Thus condition a) is satisfied with $\lambda=\bar{\lambda}$.

Since $V(\cdot)$ and its derivative are continuous and $\overline{\mathscr{D} \backslash \mathscr{N}}$ is compact (it is closed by definition and bounded since $\mathscr{D}$ is bounded) and does not contain the origin, there exists $\beta_{2}>0$ such that

$$
\frac{\left|\nabla V(x)^{\top} A_{c} x\right|}{V(x)}<\beta_{2}, \quad \forall x \in \overline{\mathscr{D} \backslash \mathscr{N}} .
$$

It follows that

$$
\dot{V}(x)<\beta_{2} V(x), \quad \forall x \in \overline{\mathscr{D} \backslash \mathscr{N}}
$$

along the trajectories of (12). From (15) and (16), and for $J \in \mathbb{N}$ big enough, then $\max \left\{\beta_{1}, \beta_{2}\right\} \leq-\ln (\lambda) / \tau_{J}=\alpha$, which implies satisfaction of $b$ ).

Theorem 2 shows that Theorem 1 is not conservative in the sense that, if there exists an exponentially decreasing Lyapunov function for the system composed by (1) and (3) which satisfies (14) and (15), then the conditions in Theorem 1 will also hold for this function. This will be used afterwards to guarantee the finite termination of the proposed algorithm to compute local Lyapunov functions and estimates of the RAO.

\section{B. Convex Conditions}

In order to obtain testable conditions, in this section we apply Theorem 1 considering $V(x)$ as a quadratic function. This will allow to express conditions a) and b) as linear matrix inequalities (LMIs) 
and therefore to formulate convex optimization problems to determine estimates of the RAO of the closed-loop system.

To deal with the saturation term present in (13), we consider the generalized sector condition proposed in [25]. For this, consider a dead-zone nonlinearity defined as follows:

$$
\phi(K x) \triangleq \operatorname{sat}(K x)-K x
$$

Lemma 1: [25] Let a matrix $G \in \mathbb{R}^{m \times n}$. The relation

$$
\phi^{\prime}(K x) T(\phi(K x)+G x) \leq 0
$$

is verified for any diagonal positive definite matrix $T \in \mathbb{R}^{m \times m}$, provided that $x$ belongs to the set

$$
H(K-G, 1) \triangleq\left\{x \in \mathbb{R}^{n}:\left|(K-G)_{(i)} x\right| \leq 1, \forall i \in \mathbb{N}_{m}\right\} .
$$

Using the deadzone function defined in (17) it follows that (13) can be re-written as

$$
y_{k}=\left(A\left(d_{j}\right)+B\left(d_{j}\right) K\right) x_{k}+B\left(d_{j}\right) \phi\left(K x_{k}\right) .
$$

Based on (18) and the conditions in Theorem 1 with a quadratic function $V(x)=x^{\prime} P x$ with $P=P^{\prime}>0$, we can now state a constructive condition to address Problem 1.

Theorem 3: If there exist a matrix $W=W^{\prime}>0, W \in \mathbb{R}^{n \times n}$, matrices $R_{j} \in \mathbb{R}^{m \times n}$ and diagonal positive definite matrices $S_{j} \in$ $\mathbb{R}^{m \times m} \forall j \in \mathbb{N}_{J}$, and scalars $\lambda \in(0,1)$ and $\alpha=-\ln (\lambda) / \tau_{J}>$ $\max \left\{2 \sigma_{\max }\left(A_{c}\right), 0\right\}$ satisfying the following LMIs

$$
\begin{gathered}
{\left[\begin{array}{ccc}
\lambda W & R_{j}^{\prime} & W A^{\prime}\left(d_{j}\right)+W K^{\prime} B^{\prime}\left(d_{j}\right) \\
\star & 2 S_{j} & S_{j} B^{\prime}\left(d_{j}\right) \\
\star & \star & W
\end{array}\right] \geq 0, \forall j \in \mathbb{N}_{J}} \\
{\left[\begin{array}{cc}
W & W K_{(i)}^{\prime}-R_{j(i)}^{\prime} \\
\star & 1
\end{array}\right] \geq 0, \forall j \in \mathbb{N}_{J}, \forall i \in \mathbb{N}_{m}} \\
W A_{c}^{\prime}+A_{c} W-\alpha W<0
\end{gathered}
$$

then, for all $x\left(0^{-}\right)=x_{0} \in \mathscr{E}\left(W^{-1}, 1\right)=\left\{x \in \mathbb{R}^{n}: x^{\prime} W^{-1} x \leq 1\right\}$, it follows that the corresponding trajectory of the sampled-data system (1)-(3), with $\delta_{k}$ satisfying (2), converges asymptotically to the origin.

Proof: Consider $V(x)=x^{\prime} P x$ with $P=W^{-1}$. By using the change of variables $G_{j}=R_{j} P$, by left and right multiplying (19) by $\operatorname{Diag}\left(P, T_{j}, I\right)$, denoting $T_{j}=S_{j}^{-1}$, by using Schur's complement and finally by left and right multiplying the resulting inequality respectively by $\left[x_{k}^{\prime}, \phi^{\prime}\left(K x_{k}\right)\right]$ and its transpose, one can conclude that (19) implies that:

$$
y_{k}^{\prime} P y_{k}-\lambda x_{k}^{\prime} P x_{k}-2 \phi^{\prime}\left(K x_{k}\right) T_{j}\left(\phi\left(K x_{k}\right)+G_{j} x_{k}\right) \leq 0,
$$

for all $j \in \mathbb{N}_{J}$ and all $x_{k} \in \mathbb{R}^{n}$, with $y_{k}$ given by (18). Thus, from Lemma 1, it follows that $V\left(y_{k}\right) \leq \lambda V\left(x_{k}\right)$ for all $y_{k} \in \mathscr{F}_{J}\left(x_{k}\right)$, provided that $x_{k} \in \bigcap_{j \in \mathbb{N} J} H\left(K-G_{j}, 1\right)$. That is, condition a) of Theorem 1 is satisfied with

$$
\mathscr{D}=\bigcap_{j \in \mathbb{N}_{J}} H\left(K-G_{j}, 1\right) .
$$

Moreover, from (21) it follows that item b) of Theorem 1 is verified with $V(x)=x^{\prime} P x$. At this point note that (21) can be verified if and only if the eigenvalues of $A_{c}$ have real part strictly smaller than $\alpha / 2$. Thus, it is necessary to consider $\alpha>\max \left\{2 \sigma_{\max }\left(A_{c}\right), 0\right\}$ in order to ensure the feasibility of inequality (21).

Hence, from Theorem 1, the sublevel set $\Omega_{1}=\mathscr{E}\left(W^{-1}, 1\right)$ associated to function $V$ is included in the RAO of system (1)-(3) provided that $\mathscr{E}\left(W^{-1}, 1\right) \subseteq \mathscr{D}$, which is guaranteed by (20), as shown next.
Applying Schur's complement to (20) and left and right multiplying the resulting inequality by $x_{k}^{\prime} P$ and $P x_{k}$ respectively, it follows that

$$
\left|\left(K-G_{j}\right)_{(i)} x_{k}\right|^{2} \leq x_{k}^{\prime} P x_{k}, \quad \forall j \in \mathbb{N}_{J}, \forall i \in \mathbb{N}_{m}, \forall x_{k} \in \mathbb{R}^{n}
$$

which ensures that $\mathscr{E}\left(W^{-1}, 1\right) \subseteq \mathscr{D}$ and concludes the proof.

Remark 3: Notice that the periodic sampling case (i.e. when $\tau_{m}=$ $\left.\tau_{M}\right)$ is obtained as a particular case with the set $\Delta_{J}$ as $\Delta_{J}=\left\{\tau_{m}\right\}=$ $\left\{\tau_{M}\right\}$. In this case condition (21) can be discarded and the LMIs (19) and (20) turn out to be the classical LMIs used for linear discretetime systems subject to input saturations when the generalized sector condition is used to deal with the saturation term (see [26, Section 3.6]).

\section{Optimization Problems}

Given the bounds $\tau_{m}$ and $\tau_{M}$ on $\delta_{k}$, we can use the conditions of Theorem 3 to compute regions of guaranteed stability for the sampled-data closed-loop system, i.e. estimates of the region of attraction of the origin. Actually, provided $x_{0} \in \mathscr{E}(P, 1)$ (with $P=$ $\left.W^{-1}\right)$, conditions of Theorem 1 guarantee that the corresponding trajectory converges asymptotically to the origin.

As pointed out in Remark 2, the region $\mathscr{E}(P, 1)$ is defined in the space of $x=\left[\begin{array}{ll}x_{p}^{\prime} & u^{\prime}\end{array}\right]^{\prime}$ but we want to define the estimate of the RAO in the $x_{p}$-hyperplane, considering the initial value of $u\left(0^{-}\right)$ as a free parameter to be determined. In fact, if the extended state $x_{0}=\left[\begin{array}{ll}x_{p, 0}^{\prime} & u\left(0^{-}\right)^{\prime}\end{array}\right]^{\prime}$ is in $\mathscr{E}\left(W^{-1}, 1\right)$, with $W$ satisfying the LMI conditions given in Theorem 3 , then $x_{k} \in \mathscr{E}\left(W^{-1}, 1\right)$ for all $k \in \mathbb{N}$ and converges asymptotically to the origin. Thus, the set of states $x_{p, 0}$, for which an input $u\left(0^{-}\right)$can be defined such that the resulting $x_{0}=\left[\begin{array}{lll}x_{p, 0}^{\prime} & u\left(0^{-}\right)^{\prime}\end{array}\right]^{\prime}$ belongs to $\mathscr{E}\left(W^{-1}, 1\right)$ is an estimate of the RAO.

Defining the following partitions of $P$ and $W=P^{-1}$ :

$$
P=\left[\begin{array}{ll}
P_{11} & P_{12} \\
P_{12}^{\prime} & P_{22}
\end{array}\right], \quad W=\left[\begin{array}{ll}
W_{11} & W_{12} \\
W_{12}^{\prime} & W_{22}
\end{array}\right]
$$

where $P_{11}, W_{11} \in \mathbb{R}^{n_{p} \times n_{p}}$ and $P_{22}, W_{22} \in \mathbb{R}^{m \times m}$ and

$$
u\left(0^{-}\right)=-P_{22}^{-1} P_{12}^{\prime} x_{p, 0}
$$

the set $\mathscr{E}\left(P_{11}-P_{12} P_{22}^{-1} P_{12}^{\prime}, 1\right) \subseteq \mathbb{R}^{n_{p}}$ results to be an estimate of the RAO. In fact, if $x_{p, 0} \in \mathscr{E}\left(P_{11}-P_{12} P_{22}^{-1} P_{12}^{\prime}, 1\right)$ and $u\left(0^{-}\right)$is as in (23) then

$$
\begin{aligned}
x_{0}^{\prime} P x_{0} & =x_{p, 0}^{\prime} P_{11} x_{p, 0}+2 x_{p, 0}^{\prime} P_{12} u\left(0^{-}\right)+u\left(0^{-}\right)^{\prime} P_{22} u\left(0^{-}\right) \\
& =x_{p, 0}^{\prime} P_{11} x_{p, 0}-x_{p, 0}^{\prime} P_{12} P_{22}^{-1} P_{12}^{\prime} x_{p, 0} \leq 1
\end{aligned}
$$

which means that $x_{0} \in \mathscr{E}(P, 1)=\mathscr{E}\left(W^{-1}, 1\right)$.

On the other hand, it can be checked that

$$
P^{-1}=W=\left[\begin{array}{cc}
Q^{-1} & -Q^{-1} P_{12} P_{22}^{-1} \\
\star & P_{22}^{-1}+P_{22}^{-1} P_{12}^{\prime} Q^{-1} P_{12} P_{22}^{-1}
\end{array}\right],
$$

where

$$
Q \triangleq P_{11}-P_{12} P_{22}^{-1} P_{12}^{\prime}
$$

is the Schur complement of $P_{22}$ with respect to $P$. That is, we have that $\mathscr{E}\left(P_{11}-P_{12} P_{22}^{-1} P_{12}^{\prime}, 1\right)=\mathscr{E}\left(W_{11}^{-1}, 1\right)$.

Thus, the idea is to maximize this "safe" set of plant initial states given by $\mathscr{E}\left(W_{11}^{-1}, 1\right)$, considering some size criterion. For instance, the maximization of the minor axis of the set can be considered through the following optimization problem:

$$
\begin{aligned}
& \max _{W, R_{j}, S_{j}, \varepsilon} \varepsilon \\
& \quad \text { subject to: } \\
& (19),(20),(21), \\
& W_{11}-\varepsilon I>0 .
\end{aligned}
$$


Other size criteria, such as the volume maximization or the maximization of the set in certain directions can also be easily considered (see [26]).

Note that problem (24) is associated to a given partition of the interval $\left[\tau_{m}, \tau_{M}\right]$. In order to find a suitable partition, the following algorithm is proposed, where the maximum value $\bar{J}$ allowed for $J$ must be chosen empirically:

Algorithm:

- Step 1: Fix $\lambda$

- Step 2: Initialize $J$

- Step 3: Compute $\tau_{J}=\frac{\tau_{M}-\tau_{m}}{J}$

- Step 4: Fix $\alpha=\frac{1}{\tau_{J}} \ln \left(\frac{1}{\lambda}\right)$ and solve (24)

- Step 5: If (24) is feasible stop.

- Step 6: If $J<\bar{J}, J \leftarrow J+1$ and go to Step 3. Otherwise increase $\lambda$ and go to Step 2 .

It is interesting to note that the algorithm provides a solution if a quadratic Lyapunov function with exponential decreasing exists. In fact, assume that there exists a quadratic Lyapunov function $V(x)=$ $x^{\prime} P x, P=P^{\prime}>0$, satisfying the assumptions of Theorem 2 (and hence also of Theorem 1) for a neighbourhood $\mathscr{D} \subseteq \mathbb{R}^{n}$ of the origin. Then LMIs (19)-(21) will have a feasible solution for $W=P^{-1}$ if the value of $\lambda$ is sufficiently close to 1 and $J$ is sufficiently large. In other words, the algorithm above has a guaranteed termination since it increases iteratively the values of $\lambda$ and $J$.

To see this, suppose that there exists $V(x)=x^{\prime} P x$ such that:

$$
V\left(x_{k+1}\right) \leq \lambda V\left(x_{k}\right), \quad \forall x_{k} \in \mathscr{D}, \forall \delta_{k} \in \Delta
$$

where $x_{k+1}$ is given by (6). Since the Lyapunov condition must hold also for $x_{k}$ in $\mathscr{D}$ close enough to the origin and, then, such that $x_{k+1}=\left(A\left(\delta_{k}\right)+B\left(\delta_{k}\right) K\right) x_{k}$, it is necessary that $\lambda P \geq(A(\delta)+$ $B(\delta) K)^{\prime} P(A(\delta)+B(\delta) K)$ holds for all $\delta \in \Delta$. As this condition must hold in particular for $\delta=d_{j}, \forall j \in \mathbb{N}_{J}$, it implies the satisfaction of (19) with $R_{j}$ and $S_{j}$ null matrices for all $j \in \mathbb{N}_{J}$.

Moreover, assume without loss of generality that $\mathscr{E}(P, 1)=\{x \in$ $\left.\mathbb{R}^{n}: x^{T} P x \leq 1\right\} \subseteq H(K, 1)$, where $H(K, 1)=\left\{x \in \mathbb{R}^{n}:\left|K_{(i)} x\right| \leq\right.$ $\left.1, \forall i \in \mathbb{N}_{m}\right\}$ is the region of linearity of the system (note that the function $V(x)$ can be scaled if necessary). Then, condition (20) is satisfied with $R_{j}=0, \forall j \in \mathbb{N}_{J}$.

At last notice that, for a fixed $\lambda, \alpha=-\ln (\lambda) / \tau_{J} \rightarrow \infty$, since $\tau_{J} \rightarrow 0$, as $J \rightarrow \infty$. Thus also inequality (21) will be satisfied for a sufficiently large $J$.

Therefore, for an appropriate choice of $\lambda$ close enough to 1 , the LMI conditions (19)-(21) are satisfied for $J$ sufficiently large, and thus the algorithm has finite termination.

Remark 4: Notice that, as pointed out in the proof of Theorem 3, a necessary condition for the feasibility of (21) is that

$$
\alpha=-\frac{\ln (\lambda)}{\tau_{J}}>\max \left\{2 \sigma_{\max }\left(A_{c}\right), 0\right\}
$$

Hence, for a fixed $\lambda$, we should have

$$
\tau_{J}<-\frac{\ln (\lambda)}{\max \left\{2 \sigma_{\max }\left(A_{c}\right), 0\right\}},
$$

which implies, from (7), that the initialization of $J$ in Step 2 of the Algorithm should satisfy:

$$
J>\left(\tau_{m}-\tau_{M}\right) \frac{\max \left\{2 \sigma_{\max }\left(A_{c}\right), 0\right\}}{\ln (\lambda)} .
$$

Remark 5: The presented method requires the computation of exponential matrices for each value $d_{j} \in \Delta_{J}$. This computation can be done in many ways, using for instance the matrix eigenvalues or its characteristic polynomial, but in either way approximation errors seem unavoidable, see [27]. The effect of these approximations should be worth of further analysis, specially when $J \rightarrow \infty$.

\section{STABILIZATION}

The LMI problem (24) can be easily adapted if we want to design a feedback gain $K$ that maximizes the set $\mathscr{E}\left(W_{11}^{-1}, 1\right)$. It suffices to perform the following change of variables:

$$
Z \triangleq K W
$$

As discussed in [28] for the periodic sampling case, the control law computed from (24), without any additional performance constraint, can lead to a large region of stability, but will in general result in a very slow behavior. Moreover, it is not fair to demand the same performance level when the control is saturated, since the system operates in open-loop in this case. Hence, an effective way of balancing performance and size of the region of attraction is to force some performance constraint only when the system operates in the linearity control region. For instance, we can add to (24) the following LMIs:

$$
\left[\begin{array}{cc}
\gamma \lambda W & W A^{\prime}\left(d_{j}\right)+Z^{\prime} B^{\prime}\left(d_{j}\right) \\
\star & W
\end{array}\right] \geq 0, \forall j \in \mathbb{N}_{J}
$$

where $0<\gamma<1$ is a parameter fixed a priori. Note that constraints (26) and (21) impose that $V\left(x_{k+1}\right)<\gamma V\left(x_{k}\right)$ when the control is not saturated, i.e. they ensure a more stringent exponential decay convergence rate for the operation of the system in the linearity region.

Remark 6: If we consider $K_{u}=0$, we can also perform the substitution (25), but in this case, to preserve linearity, $W$ must be block diagonal, i.e. $W_{12}=0$, which implies that $Z=\left[\begin{array}{lll}K_{p} W_{11} & 0\end{array}\right]$.

Remark 7: It should be noticed that it is not possible to apply the change of variable in (25), when the conditions in [21] are considered, making rather difficult to address the design problem. This comes from the fact that the difference inclusion considered in [21] is different from (8).

\section{Numerical EXAMPLE}

Consider system (1)-(3) with the following matrices [26]:

$$
\begin{aligned}
A_{p} & =\left[\begin{array}{ll}
0 & 1 \\
1 & 0
\end{array}\right], B_{p}=\left[\begin{array}{c}
0 \\
-5
\end{array}\right], \\
K_{p} & =\left[\begin{array}{ll}
2.6 & 1.4
\end{array}\right], K_{u}=\left[\begin{array}{l}
0
\end{array}\right],
\end{aligned}
$$

with the interval of admissible intersampling times given by $\Delta=$ $[0.05,0.1]$.

In this case, for $\lambda=0.98$ a feasible solution for the optimization problem (24) is obtained with a partition of $\Delta$ in 30 sub-intervals (i.e. $J=30$ ), leading to:

$$
W^{-1}=\left[\begin{array}{ccc}
0.2580 & 0.1012 & -0.0204 \\
0.1012 & 0.0868 & -0.0019 \\
-0.0204 & -0.0019 & 0.0211
\end{array}\right], \varepsilon=3.4785
$$

Considering now the stabilization problem described in Section $\mathrm{V}$ with $\gamma=0.9$ and the same values for $\lambda$ and $J$, we obtain

$$
K=\left[\begin{array}{lll}
1.13 & 0.94 & 0.008
\end{array}\right] \text {. }
$$

and the region of attraction estimate given by

$$
W^{-1}=\left[\begin{array}{ccc}
0.0612 & 0.0562 & -0.0037 \\
0.0562 & 0.0608 & -0.0012 \\
-0.0037 & -0.0012 & 0.0127
\end{array}\right], \varepsilon=8.60 .
$$

Figure 2 shows the resulting estimates of the region of attraction of the origin. The ellipsoid obtained with the designed $K$ is indeed larger than the original one, as expected. For comparison purposes, we also 


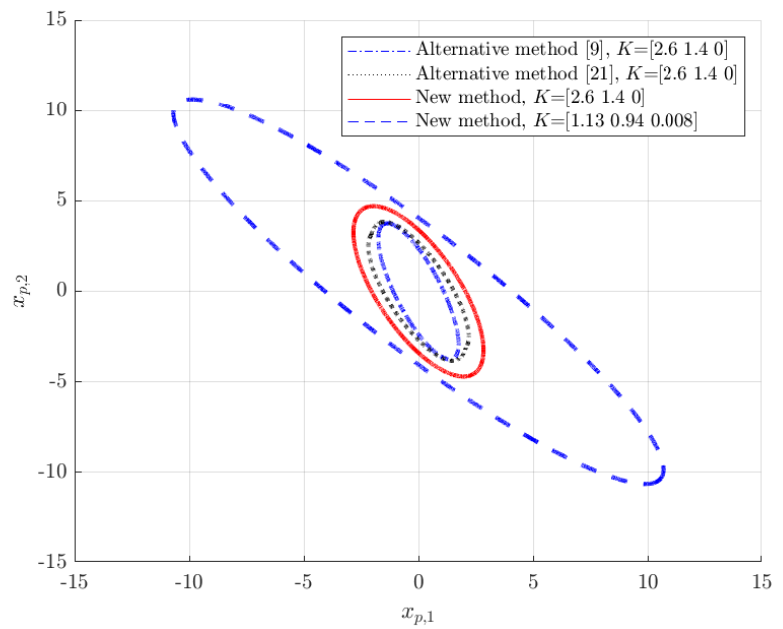

Fig. 2. Estimates of the region of attraction of the origin.

plotted the estimate of the RAO obtained with the method proposed in [21], which corresponds to the sublevel set of a piecewise quadratic function, and with the looped-functional approach proposed in [9], which is an ellipsoidal domain. As it can be observed, the method proposed here resulted in a larger domain for the same value of $K$.

In Figure 3, several trajectories with $x_{p, 0}$ at the boundary of the region $\mathscr{E}\left(W_{11}^{-1}, 1\right)$ and $u\left(0^{-}\right)$chosen as in (23) considering $K$ given in (27) and $\delta_{k}$ randomly chosen in the interval $[0.05,0.1]$ are shown. As expected, the convergence of the trajectories to the origin is ensured, which shows that $\mathscr{E}\left(W_{11}^{-1}, 1\right)$ is indeed included in its region of attraction.

Remark 8: Concerning the comparison with other approaches, it has to be noticed that, in this numerical example, our method provides a better estimate of the region of attraction of the origin, but there is no formal guarantee that this will be always the case. Furthermore, the method presented in this work is particularly suitable to deal with both the stability analysis and control synthesis problems. For instance, with the looped-functional approach [9], to obtain tractable synthesis conditions in LMI form, the Finsler's Lemma with a particular structure of the multipliers (which is an important source of conservatism) and a fixed parameter, that has to be manually tuned, are employed. On the other hand, due to the exponential dependence of $A(\delta)=e^{A_{c}} \delta_{A_{r}}$ and $B(\delta)=e^{A_{c} \delta} B_{r}$, which appear in (19), on $A_{c}$, it is not trivial with our method to cope with uncertainties in the system matrices $A_{p}$ and $B_{p}$, that are easily manageable in the loopedfunctional framework. Actually, establishing a formal comparison between our method and the looped-functional one might be rather difficult as the tools and conditions are significantly different.

\section{CONCLUSIONS}

In this paper a new approach has been proposed to deal with sampled-data controlled linear systems under aperiodic sampling and input saturation which, unlike the method presented in [21], can be used not only for the stability analysis but also for the design of a stabilizing control law. By means of a numerical example, we have shown that the proposed approach can lead to a considerable conservatism reduction, in terms of estimate of RAO, when compared to other ones reported in the literature.

The approach can be easily extended to cope with other continuous input nonlinearities, in particular sector-bounded ones. Note that in this case it suffices to replace the saturation function in model (4)

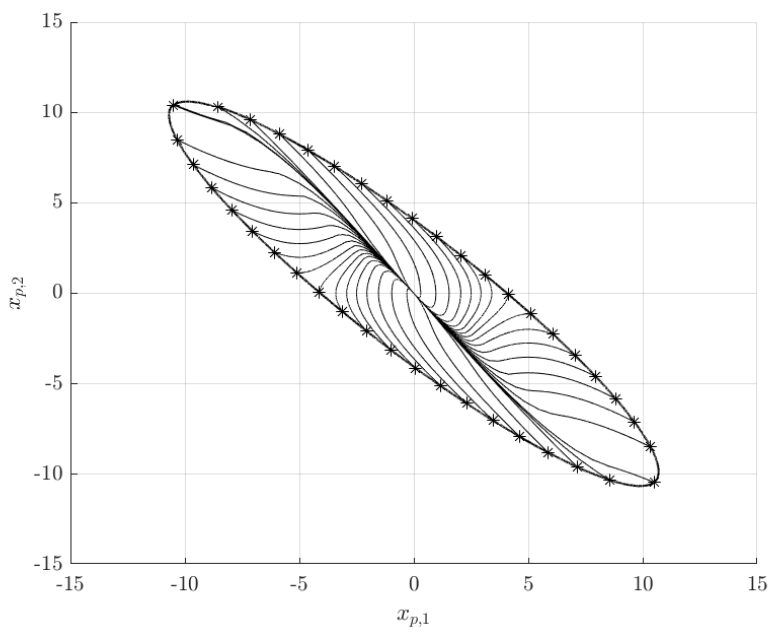

Fig. 3. Trajectories starting at the boundary of $\mathscr{E}\left(W_{11}^{-1}, 1\right)$.

by the new nonlinearity. Similar conditions to the one in Theorem 3 can therefore be derived using classical sector conditions.

As a future work, we consider to explore the use of not only quadratic but more general Lyapunov functions as, for instance, polyhedral ones.

\section{RefERENCES}

[1] K. J. Aström and B. Wittenmark, Computer Controlled Systems. Theory and Design, ser. Information and System Sciences Series. Prentice-Hall, 1984.

[2] T. Chen and B. Francis, Optimal sampled-data control systems. Berlin, Germany: Springer-Verlag, 1995.

[3] D. Nesić and A. Teel, "Sampled-data control of nonlinear systems: An overview of recent results," in Perspectives in robust control. Lecture Notes in Control and Information Sciences, vol. 268. London: Springer, 2001.

[4] S. Monaco and D. Normand-Cyrot, "Issues on nonlinear digital control," European Journal of Control, vol. 7, no. 2, pp. 160-177, 2001.

[5] W. Zhang, M. Branicky, and S. Phillips, "Stability of networked control systems," IEEE Control Systems Magazine, vol. 21(1), pp. 84-99, 2001.

[6] E. Fridman, "A refined input delay approach to sampled-data control," Automatica, vol. 46, no. 2, pp. 421-427, 2010.

[7] K. Liu and E. Fridman, "Wirtinger's inequality and Lyapunov-based sampled-data stabilization," Automatica, vol. 48, no. 1, pp. $102-108$, 2012.

[8] A. Seuret, "A novel stability analysis of linear systems under asynchronous samplings," Automatica, vol. 48, no. 1, pp. 177-182, 2012.

[9] A. Seuret and J. M. Gomes da Silva Jr., "Taking into account period variations and actuator saturation in sampled-data systems," Systems \& Control Letters, vol. 61, pp. 1286-1293, 2012.

[10] L. Hetel, J. Daafouz, J. Richard, and M. Jungers, "Delay-dependent sampled-data control based on delay estimates," Systems \& Control Letters, vol. 60, no. 2, pp. 146 - 150, 2011.

[11] M. Fiacchini and I.-C. Morărescu, "Constructive necessary and sufficient condition for the stability of quasi-periodic linear impulsive systems," IEEE Transactions on Automatic Control, vol. 61, no. 9, pp. 2512-2517, 2016.

[12] M. Cloosterman, L. Hetel, N. V. D. Wouw, W. Heemels, J. Daafouz, and H. Nijmeijer, "Controller synthesis for networked control systems," Automatica, vol. 46, no. 10, pp. 1584-1594, 2010.

[13] W. Lombardi, S. Olaru, S.-I. Niculescu, and L. Hetel, "A predictive control scheme for systems with variable time-delay," International Journal of Control, vol. 85, no. 7, pp. 915-932, 2012.

[14] H. Fujioka, "A discrete-time approach to stability analysis of systems with aperiodic sample-and-hold devices," IEEE Transactions on Automatic Control, vol. 54, no. 10, pp. 2440-2445, 2009. 
[15] H. Fujioka and Y. Oishi, "A switched Lyapunov function approach to stability analysis of non-uniformly sampled-data systems with robust LMI techniques," in 2011 8th Asian Control Conference (ASCC), 2011, pp. $1487-1491$.

[16] C.-Y. Kao and H. Fujioka, "On stability of systems with aperiodic sampling devices," IEEE Trans. on Automatic Control, vol. 28, no. 3, pp. 2085-2090, 2013.

[17] C. Briat, "Convex conditions for robust stability analysis and stabilization of linear aperiodic impulsive and sampled-data systems under dwelltime constraints," Automatica, vol. 49, no. 11, pp. 3449-3457, 2013.

[18] P. Naghshtabrizi, J. Hespanha, and A. Teel, "Exponential stability of impulsive systems with application to uncertain sampled-data systems," Systems \& Control Letters, vol. 57, no. 5, pp. 378-385, 2008.

[19] L. Hetel, C. Fiter, H. Omran, A. Seuret, E. Fridman, J.-P. Richard, and S. I. Niculescu, "Recent developments on the stability of systems with aperiodic sampling: An overview," Automatica, vol. 76, pp. 309 - 335, 2017.

[20] J. M. Gomes da Silva Jr., I. Queinnec, A. Seuret, and S. Tarbouriech, "Regional stability analysis of discrete-time dynamic output feedback under aperiodic sampling and input saturation," IEEE Transactions on Automatic Control, vol. 61, no. 12, pp. 4176-4182, 2016.

[21] M. Fiacchini and J. M. Gomes da Silva Jr., "Stability of sampled-data control systems under aperiodic sampling and input saturation," in 2018 IEEE Conference on Decision and Control (CDC), 2018, pp. 6644-6649.

[22] M. Cloosterman, N. V. D. Wouw, W. Heemels, and H. Nijmeijer, "Stability of networked control systems with uncertain time-varying delays," IEEE Trans. on Automatic Control, vol. 54, no. 7, pp. 1575$1580,2009$.

[23] L. Hetel, J. Daafouz, S. Tarbouriech, and C. Prieur, "Stabilization of linear impulsive systems through a nearly-periodic reset," Nonlinear Analysis: Hybrid Systems, vol. 7, no. 1, pp. 4-15, 2013.

[24] H. J. Sussmann, S. D. Sontag, and Y. Yang, "A general result on the stabilization of linear systems using bounded controls," IEEE Transactions on Automatic Control, vol. 39, no. 12, pp. 2411-2425, 1994.

[25] J. M. Gomes da Silva Jr. and S. Tarbouriech, "Anti-windup design with guaranteed region of stability: an LMI-based approach," IEEE Trans. on Automatic Control, vol. 50, no. 1, pp. 106-111, 2005.

[26] S. Tarbouriech, G. Garcia, J. M. Gomes da Silva Jr., and I. Queinnec, Stability and Stabilization of Linear Systems with Saturating Actuators. Springer, 2011.

[27] C. Moler and C. Van Loan, "Nineteen dubious ways to compute the exponential of a matrix, twenty-five years later," SIAM Review, vol. 45(1), pp. 3-49, 2003.

[28] J. M. Gomes da Silva Jr. and S. Tarbouriech, "Local stabilization of discrete-time linear systems with saturating controls: an LMI-based approach," IEEE Trans. on Automatic Control, vol. 46, no. 1, pp. 119$125,2001$. 